\title{
Process intensification by coupling the Joule effect with pervaporation and sweeping gas membrane distillation
}

\author{
S. Shukla ${ }^{a}$, J.P. Méricq ${ }^{a}$, M.P. Belleville ${ }^{\mathrm{a}}$, N. Hengl ${ }^{\mathrm{d}}$, N.E. Benes ${ }^{\mathrm{b}}$, I. Vankelecom ${ }^{\mathrm{c}}$, \\ J. Sanchez Marcano ${ }^{\text {a,* }}$ \\ ${ }^{a}$ Institut Européen des Membranes, Université de Montpellier, CC 047, Montpellier Cedex 5 34095, France \\ ${ }^{\mathrm{b}}$ Membrane Science and Technology Cluster, Department of Science and Technology, Mesa + Institute for Nanotechnology, Universiteit Twente, 7500 AE Enschede, The \\ Netherlands \\ c Centre for Surface Chemistry and Catalysis, KU Leuven, PO Box 2461, Celestijnenlaan 200F, 3001 Leuven, Belgium \\ ${ }^{\mathrm{d}}$ Université Grenoble Alpes, LRP, F-38000 Grenoble, France
}

A R T I C L E I N F O

\section{Keywords:}

Process intensification

Metallic membranes

Joule effect

SGMD

Pervaporation

\begin{abstract}
A B S T R A C T
This work concerns the intensification of membrane processes by coupling the Joule effect with two membrane processes: pervaporation and sweeping gas membrane distillation. For this purpose, conducting metallic hollow fibers impregnated or coated with polydimethyl siloxane were simultaneously used as membrane and heating electric resistance. The application of an electrical potential resulted in an enhancement of $40 \%$ of the water vapor permeate flux in sweep gas membrane distillation. However, the flux enhancement is the result not only of the heating on the membrane vicinity but also on the enhancement of the feed temperature. In the case of pervaporation of aqueous ethanol solutions (20\%), the direct heating of fibers allowed improving by $100 \%$ the ethanol permeate flux while increasing the process selectivity.
\end{abstract}

\section{Introduction}

The sweeping gas membrane distillation process (SGMD) is a hybrid evaporation-concentration or distillation process carried out in membrane contactors with hydrophobic macroporous membranes. In this process, the membrane is not selective but it is only the support of a liquid-vapor interface located at the pore inlet between an aqueous solution on the membrane feed side and a flow of dry air on the membrane permeate side. SGMD involves evaporation of water from the hot feed solution, and transfer of the vapor molecules through the non-wetting pores of the hydrophobic membrane. This transport is driven by the water vapor partial pressure difference between feed and permeate sides of the membrane. Subsequently, the permeated molecules are carried by an inert cold sweeping gas and finally collected outside the membrane module. In the case that SGMD is used as distillation process, the permeate flow is then cooled down in order to condense the evaporated water. The SGMD [1-3] process is less often studied compared to other membrane distillation configurations, like direct contact membrane distillation (DCMD), due to the small volume of permeate vapors generated and the need of external condensers. However, SGMD presents lower internal heat losses by conduction compared to DCMD and higher permeate fluxes in comparison to air gap membrane distillation (AGMD) due to the non-stationary sweeping gas. This makes SGMD a technology with promising future perspectives.

In pervaporation, a liquid feed mixture contacts one side of a membrane while the permeate is removed as a vapor from the other side. The membrane can be hydrophobic or hydrophilic, depending on the targeted application. Transport through the membrane is induced by the difference in vapor partial pressure between the feed solution and the permeate vapor. This vapor pressure difference can be maintained in various ways. In general, at lab scale vacuum is maintained on the permeate side using a vacuum pump, while the permeate vacuum in industry is most economically generated by cooling and condensing the permeate vapor spontaneously creating a partial vacuum. The third possible way is to sweep the permeate side of the membrane with a counter-current flow of carrier gas. Carrier gas can then be cooled down to condense and recover permeate vapor [4].

Separation by pervaporation is based on the solution-diffusion mechanism of transport. The mass transport across the permselective membrane involves three successive steps: (a) upstream partitioning of the feed components between the flowing liquid mixture and the swollen upstream surface layer of the membrane according to their affinity for membrane material, (b) diffusion of the selectively absorbed components through the membrane, and (c) desorption of these

\footnotetext{
* Corresponding author at: Institut Européen des Membranes, Université de Montpellier, CC 047, Montpellier Cedex 534095 , France.

E-mail address: Jose.Sanchez-Marcano@umontpellier.fr (J. Sanchez Marcano).
} 
components at the downstream surface of this membrane [5]. Dehydration of solvents, removal of volatile organic components from water (water purification) and organic/organic separation as an alternative to conventional distillation are the most common applications of pervaporation process.

Classified under inorganic membranes, metallic hollow fiber membranes exhibit superior mechanical and thermal stabilities in comparison to polymeric ones. In addition, their metallic nature bestows thermal and electrical conduction properties and higher mechanical strength than other inorganic membranes like ceramic ones. Furthermore, their electrical resistivity makes metals interesting materials for direct heating of the membrane by the Joule effect. This way, the enthalpy of evaporation in SGMD and pervaporation can be supplied directly at the membrane surface where the evaporation takes place. This direct heating can thus limit the temperature decrease on the membrane feed side (the so-called temperature polarization) and reduce the subsequent mass transfer decrease due to this phenomenon.

Moreover, such localized heating of membranes can be very interesting for the concentration or separation of thermal sensitive solutions, because the effectiveness of the global process can be maintained without a too important heating of the whole feed solution. This principle was recently successfully applied in some membrane fields, i.e., pervaporation and solvent resistant nanofiltration, using the photothermal effect [6-8]. In SGMD or membrane evaporation, Hengl et al. [9-11] have previously demonstrated that temperature polarization can be minimized and water permeate fluxes can be increased by using flatsheet metallic membranes and heating them by infrared rays or by the Joule effect using them as electrical resistance. However, in this previous work, the process was limited by the hydrodynamic conditions that were imposed because of the design of the module and by the structural membrane properties (thickness, pore size, hydrophobicity). Furthermore, flat-sheet membranes have a very low packing density that increases their footprint in comparison to hollow fiber membranes.

In more recent work [12] a multidisciplinary approach was presented that includes the development of macro-porous metallic hollow fibers giving hydrophobic properties to the surface with polydimethylsiloxane (PDMS), and finally the study of the SGMD process. Experiments were conducted with pure water, and effects of different process parameters on permeate flux, such as sweeping gas velocity and feed temperature, were investigated. A second set of experiments was conducted with sucrose solutions of different concentrations in order to assess the possibility of using this new metallic membrane system for the concentration of thermally sensitive/biological solutions.

Only few existing studies have reported alternative approaches to supply energy necessary for evaporation during pervaporation. Applying microwave heating for hydrophilic pervaporation through PVA-PAN cross-linked membrane was reported [13], as well as pervaporation using hollow fiber membranes in the electromagnetic microwave field [14]. Localized membrane heating was also established by means of photo thermal heating of silver nanoparticles dispersed in the PDMS layer supported on PVDF [7]. In this perspective, using metallic membranes could give immense opportunity to bring energy required for evaporation directly to the membrane surface in the course of the pervaporation process. This approach is both less energy intensive and is also of interest in applications such as recovery of valuable heat sensitive products or aroma compounds. In our study, the possibility of doing this is demonstrated by carrying out preliminary hydrophobic pervaporation experiments using ethanol-water feed.

This work presents the preliminary results of an innovative and multidisciplinary approach starting from the selection and the modification of metallic hollow fibers by a hydrophobic treatment with PDMS and the use of such metallic membranes as electric resistance in order to improve SGMD and pervaporation performance by diminishing the effects of the temperature polarization. In the case of SGMD, experiments were carried out in a module with a bundle of hydrophobic macroporous metallic hollow fibers. A module with a unique metallic fiber covered with a dense layer of PDMS was used to study the pervaporation of aqueous solutions of ethanol.

\section{Experimental}

\subsection{Materials}

Stainless steel (SS) powder (316L) with particle size of $4.17 \mu \mathrm{m}$ (D50 by the manufacturer) was purchased from Epson Atmix Corporation (Japan). Polyetherimide (PEI), N-methylpyrrolidone (NMP, $99.5 \mathrm{wt} \%$ ), polyvinylpyrrolidone (PVP K95) were bought from Aldrich. The stainless steel powder and PEI were dried before use; all other chemicals were used without further treatment. Polydimethylsiloxane (PDMS)-615 kit (General Electric) consisted on a vinyl terminated pre-polymer (RTV-A) and a platinum (Pt)-catalyzed cross-linker (RTV-B) containing a polyhydrosilane component. Ethanol ( $\geq 99.8 \%$ ) and hexane ( $\geq 97 \%$ ) were also purchased from Sigma Aldrich.

\subsection{Methods}

\subsubsection{Preparation of hydrophobic stainless steel hollow fiber membranes} for SGMD and pervaporation

Hydrophobic metallic hollow fibers for the SGMD were prepared as described in previous work [12]. They were manufactured through the non-solvent induced phase separation (NIPS) technique using SS particles $(68 \mathrm{wt} \%$ ) dispersed in $\mathrm{N}$-methylpyrrolidone (25 wt \%). After homogenization and outgassing the spinning dope was pressurized with nitrogen from a feed vessel through an spinneret, while simultaneously de-ionized water was pumped through the inner tube of the spinneret. The hollow fibers leaving the spinneret passed through brief air gap before a free fall into the external coagulant bath composed of tap water. The whole process was carried out at controlled room temperature of $269^{\circ} \mathrm{C} \mathrm{K}$. Hollow fibers precursors (green fibers) were dried and stretched under ambient conditions for overnight. Then the fibers were fired at $1348{ }^{\circ} \mathrm{C}$ under nitrogen. After cleaning and drying, native fired stainless steel fibers were impregnated with a $2 \%$ of PDMS prepolymer and cross-linker in a 10:1 weight ratio in hexane before being cured at $373 \mathrm{~K}$ overnight to evaporate the solvent and to complete the crosslinking reaction. The metallic grains forming the fibers were then covered through all membrane thickness with a very thin layer of PDMS that gives it excellent hydrophobic surface properties while maintaining an important macro-porosity as required for SGMD process. The characterizations of these membranes in terms of hydrophobic properties (water pressure intrusion and contact angle) have been reported in a previous work [12].

Pervaporation membranes were prepared using the same native fired stainless steel fibers [12], but their external surface was covered with a fine dense layer of PDMS without intrusion into the pores. For this purpose, the pre-polymer and cross-linker solution in hexane was prepared in a similar ratio as above, but at a concentration of $10 \%$ (M1) or $20 \%$ (M2). The cross-linking reaction was initiated by placing the solution into a water bath maintained at $60{ }^{\circ} \mathrm{C}$ with constant stirring for $2 \mathrm{~h}$. This initial cross-linking time is important in order to obtain PDMS solutions with required viscosity [15]. Before coating hollow fiber supports, they were immersed in hexane for $4 \mathrm{~h}$ to saturate the pores with the solvent in order to prevent the intrusion of the coating solution into the porosity. Both open sides of the supports were plugged with a paste and immersed into a pre-crosslinked PDMS solution for $5 \mathrm{~min}$. The supports were then dried at room temperature for $10 \mathrm{~min}$ followed by curing at $110{ }^{\circ} \mathrm{C}$ at least for $1 \mathrm{~h}$ to complete the cross-linking. This method allows having a thin dense PDMS layer on the external surface of the fibers without intrusion inside the pores.

\subsubsection{Characterization of the membranes}

The total pore volume, the mean pore diameter and the percent 
porosity of modified and unmodified hollow fiber membrane were measured with a Micromeritics ${ }^{\circledR}$ AutoPore IV 9500. The structure and morphology of the hollow fiber membranes were observed under SEM (Hitachi S48000). For this purpose, hollow fiber membranes were broken in liquid nitrogen to observe cross-section morphology.

\subsection{Equipment and implementation}

\subsubsection{SGMD pilot}

The original pilot unit for SGMD experiments has already been described in detail in a previous work [12]. It consists essentially in a vertically mounted hollow fiber membrane module (1) which forms the core of the system: it was composed of 25 vertically arranged stainlesssteel hollow fibers (treated with $2 \%$ of PDMS). Each fiber had a length of $1.510^{-1} \mathrm{~m}$, an inner diameter of $1.110^{-3} \mathrm{~m}$ and an outer diameter of $1.610^{-3} \mathrm{~m}$. The counter-current mode was chosen to maintain the partial pressure gradient constant all along the length of the fibers. This would eventually help to achieve higher flux values. Every experiment was carried out for fixed duration of $120 \mathrm{~min}$ to a maximum period of $240 \mathrm{~min}$. Temperature, pressure and relative humidity were recorded every five minutes. The reported values of water vapor flux were calculated under steady state conditions which were reached approximately $30 \mathrm{~min}$ after the start of each run. The calculations of water vapor flux were made considering arbitrarily the lumen side of membranes. The total area of the lumen side was of $1.410^{-2} \mathrm{~m}^{2}$. The configuration used was in countercurrent: water was fed in the shell side whereas a flow of dry air was fed inside the fibers. The difference of relative humidity measured between the input and output of the air flow was directly related with the water vapor flow as previously explained [12]. In this study the original pilot unit was modified for membrane heating by Joule effect. It was performed by applying a potential difference across the two ends of the stainless steel fibers. In order to convey homogeneous supply of electricity to all the fibers, two stainless steel plates ( $1 \mathrm{~mm}$ thick) with 25 holes homogenously located were placed at each module ends and $1 \mathrm{~mm}$ of each fiber end was introduced in each hole. Conductive glue was filled to fill the voids between fibers and the metallic plates and to establish good electrical contact. Two electrical wires were soldered at the center of each metallic plate, and connected to the positive and negative potential electrodes of low of voltage DC generator (Française d'Instrumentation, 3030D). To avoid the electrical heating of the water by the metallic plates and to ensure the electrical insulation the fibers passes through two PVC plates placed between the inner side of the module (shell side) and the conducting metallic plates. The pilot unit is shown in Fig. 1.

The electric power supplied to the membranes was controlled by regulating the voltage applied. Experiments were carried out at low voltage to avoid risks of electric shock and prevent the damage of the membrane. The energy $P_{\text {electric }}(\mathrm{W})$ delivered to the membrane was calculated with the current $I(\mathrm{~A})$ and the voltage $U(\mathrm{~V})$ imposed between both electrodes:
$P_{\text {electric }}=U . I$

Different values of current intensity were applied $(I=0 ; 2$ and $3 \mathrm{~A})$ for a voltage of $4 \mathrm{~V}$, it corresponds to 0,8 and $12 \mathrm{~W}$ of energy delivered.

\subsubsection{Pervaporation}

The pervaporation module and pilot unit, with membrane heating by Joule effect, is presented in Fig. 2. The membrane module is a transparent cylindrical glass tube containing a single hollow fiber (length of $1310^{-2}-1410^{-2} \mathrm{~m}$ and a total effective area of $310^{-4}-4$ $10^{-4} \mathrm{~m}^{2}$ ) mounted vertically with the help of Swagelok stainless steel fittings clamped over a supporting column. The ethanol/water feed (2-20 wt\%) in the feed tank was maintained at room temperature $\left(20 \pm 2{ }^{\circ} \mathrm{C}\right)$ and mixed over a magnetic stirrer (IKA-RTC classic). Feed was continuously circulated at a flowrate of $30 \mathrm{~mL} \mathrm{~min}^{-1}$ and atmospheric pressure from the feed tank to the shell-side of the module (bottom-top circulation) by a peristaltic pump (BT100-1L from Longer Pump) and was then re-circulated back to the feed tank. Bottom entrance of the fiber is connected to a vacuum pump (purchased from Edwards) to maintain a pressure of $80 \mathrm{mbar}$, measured by a pressure gauge. Permeate vapor was condensed and collected thanks to a liquid nitrogen cold trap between the module and the vacuum pump. For each time, permeate weight $W$ was measured and permeate sample was analyzed using a refractometer (ATAGO RX-7000 $\alpha$ ). Feed was also analyzed with the refractometer. Both ends of the fiber are in contact with metallic tubing to establish electrical contact. As described above for the SGMD experiments (see Section 2.3.1), both metallic ends of the membranes were connected to the positive and negative potentials electrodes of the low voltage DC generator. In all pervaporation experiments with heating a voltage of $2.5 \mathrm{~V}$ and an intensity of $4 \mathrm{~A}$ were applied, it means an energy delivered of $10 \mathrm{~W}$.

Each experiment was performed during $2 \mathrm{~h}$ to equilibrate the system and then permeate vapor was collected for $12-13 \mathrm{~h}$. The results are an average of at least three measurements. For the experiment with membrane heating by Joule effect, the current was applied after the initial two $\mathrm{h}$ and permeate collection started $30 \mathrm{~min}$ after. Change in temperature along the membrane after applying electricity was recorded at every $30 \mathrm{~min}$ interval with help of laser-based non-contact thermometer (Testo 845).

The pervaporation efficiency of the membranes was evaluated in terms of flux and separation factor. Water evaporation flux $J$ $\left(\mathrm{kg} \mathrm{h}^{-1} \mathrm{~m}^{-2}\right)$ was calculated as:

$J=\frac{W}{t(\pi d l)}$

where $W$ is the mass of collected permeate, $t$ is the permeate collection time, $d$ and $l$ are the inner diameter and length of the fiber respectively.

The separation $\alpha$ was calculated using the following equation,

$\alpha=\frac{y /(1-y)}{x /(1-x)}$

where, $y$ and $x$ are respectively the weight fractions of the components

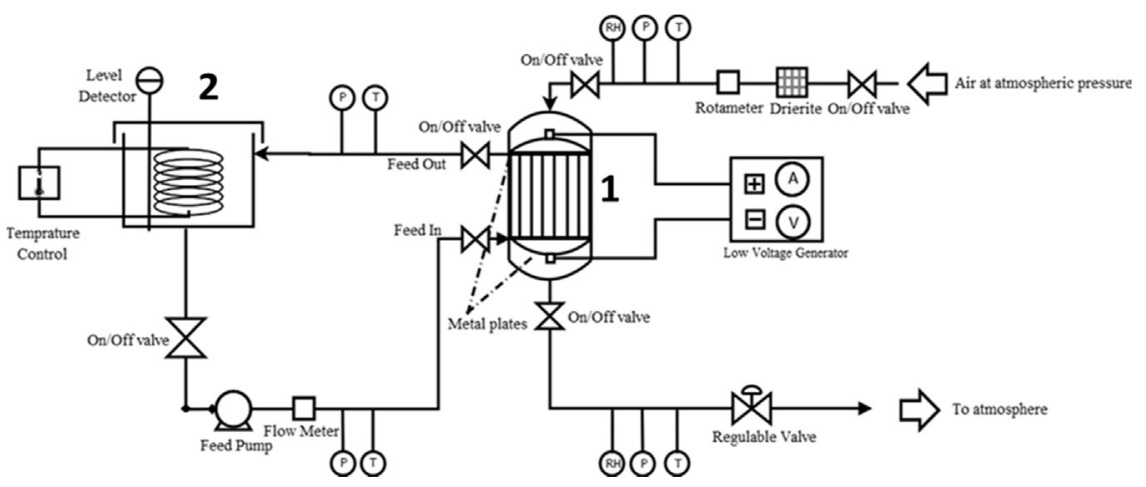

Fig. 1. Schematic of SGMD pilot unit. (1) hollow fiber membrane module, pump, (2) feed tank with heat exchanger, temperature sensors $(\mathbf{T})$, pressure sensors $(\mathbf{P})$, relative humidity sensors $(\mathbf{R H})$. 


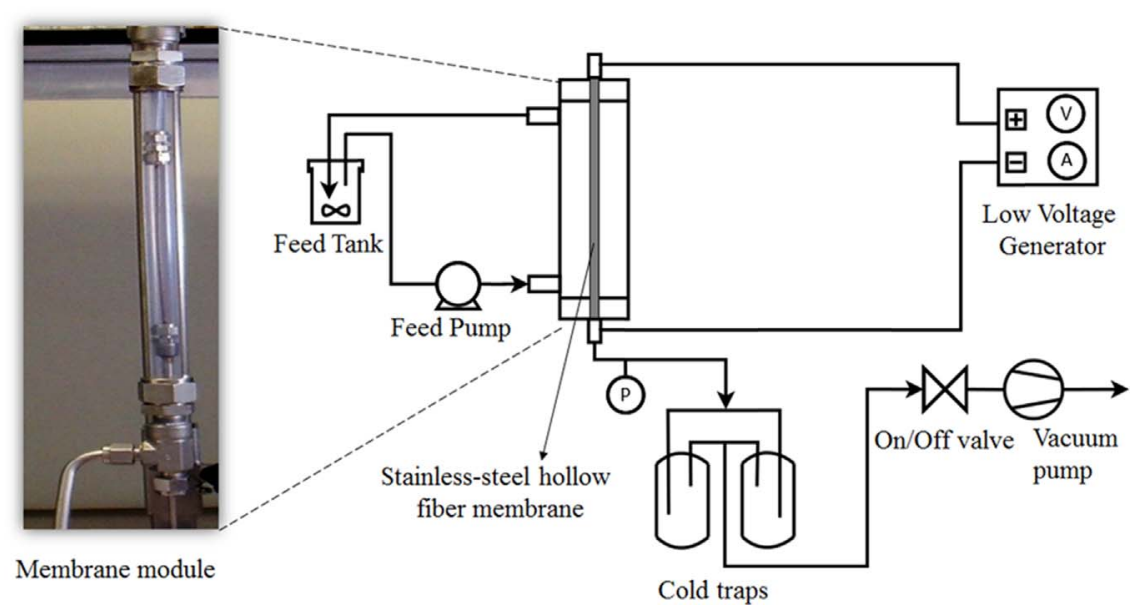

Fig. 2. Schematic of pervaporation pilot unit.

in the permeate and feed, respectively.

\section{Results and discussion}

\subsection{Membranes for SGMD and pervaporation}

Metallic hollow fiber membranes for SGMD were coated with a $2 \%$ PDMS solution described in previous work [12]. As explained above, the PDMS only coated the membrane grains with a very thin layer keeping the porosity open and without modifying the main structural characteristics (Table 1). These membranes presented good hydrophobic properties (contact angle of $134^{\circ}$ and a water intrusion pressure of $3.510^{4} \mathrm{~Pa}$ [12]). Pervaporation membranes M1 and M2 consisted of a very thin layer of dense PDMS on the outer surface of the fibers and preserved the structural characteristics of the native stainless steel supports. The main characteristics of the membranes are reported in Table 1 together with the raw membrane characteristics.

The porosity of the hollow fiber membranes remains in the same range before and after modification. The small deviations in values can be assigned to differing sample weights during measurement to which mercury porosimetry is known to be highly sensitive. The average diameter of the pores is $3.0-3.3 \mu \mathrm{m}$ for unmodified membranes, remaining unchanged after modification with the $2 \%$ PDMS solution. Surface modification thus increase membrane hydrophobicity while it induces nearly no change in the membrane structural properties.

SGMD and pervaporation fibers were compared by scanning SEM. Fig. 3 presents the comparison between the cross-section of raw membranes (without PDMS) and treated membrane used for SGMD and pervaporation.

After PDMS impregnation of SGMD membranes (Fig. 3B), no formation of polymer layer on the surface is observed. Therefore, modification only forms a monolayer over grains of stainless-steel particles that constitute the membrane structure. This result confirms the fact that modified and unmodified membranes present the same structural parameters (Table 1). In the case of pervaporation membranes
(Fig. 3C), one can notice a dense uniform layer formed on the surface. This layer covers all the pores (SEM image of the surface not presented here). The thickness of this layer, as estimated from cross section SEM image (Fig. 3D), is about 10-12 $\mu \mathrm{m}$ (membrane M2 in Table 1).

\subsection{Sweeping gas membrane distillation}

In previous work [12], the SGMD process was studied and the influence of different parameters like feed temperature, Reynolds number of water $\left(R e_{\text {water }}\right)$ and air flux $\left(R e_{\text {air }}\right)$ on the water evaporation rate was determined. In this work, comparative results are presented using the Joule effect under the same experimental conditions.

Experiments were carried out by electrically heating 2\% PDMS coated metallic hollow fiber membranes with circulation of pure water $\left(R e_{\text {water }}=145\right)$ and with a constant flow of sweeping gas $\left(R e_{\text {air }}=\right.$ 1092). During the experiment, the applied current and the feed temperatures were varied. The maximum set value for the feed temperature was $40{ }^{\circ} \mathrm{C}$ because this process is targeted to be used for thermally sensitive solutions. Fig. 4 shows a comparison of the water evaporation fluxes obtained with and without electrical heating. At a feed temperature of $25^{\circ} \mathrm{C}$ (Fig. 4A), the water evaporation flux increased from $5.910^{-2} \mathrm{~kg} \mathrm{~h}^{-1} \mathrm{~m}^{-2}$ (at $I=0 \mathrm{~A}$ ) to $7.510^{-2}$ and 8.3 $10^{-2} \mathrm{~kg} \mathrm{~h}^{-1} \mathrm{~m}^{-2}$ at an electrical current of 2.3 and $3.1 \mathrm{~A}$ respectively. These values correspond to flux enhancement factors of 1.25 and 1.4, confirming the improvement of water evaporation rates due to the membrane heating. The flux enhancement factor was calculated by the ratio of improved flux after applying electricity to the initial flux (without current) and corresponds to an applied power of 7.3 and $11.7 \mathrm{~W}$ respectively.

In SGMD, water evaporation at the membrane pore inlet induces a decrease of the local temperature (because of the water vaporization enthalpy). The temperature at the membrane surface is thus lower than the temperature in the feed which decreases permeate flux performances: it is the temperature polarization phenomenon. Using membrane direct heating, the decrease of membrane temperature due to the

Table 1

Structural parameters of membranes before and after modification with PDMS

\begin{tabular}{|c|c|c|c|c|}
\hline Properties & Native SS hollow fibers & SGMD fibers modified with $2 \%$ PDMS & Pervaporation fibers ${ }^{\mathrm{a}} \mathrm{M} 1$ & Pervaporation fibers $^{\mathrm{a}} \mathrm{M} 2$ \\
\hline Length $(\mathrm{mm})$ & 150 & 150 & 140 & 140 \\
\hline Wall thickness $(\mu \mathrm{m} \pm 20)$ & 240 & 220 & 220 & 220 \\
\hline Outer diameter/inner diameter (mm) & $1.6-1.5 / 1.3-1.2$ & $1.5-1.4 / 1.2-1.1$ & $1.5-1.4 / 1.2-1.1$ & $1.5-1.4 / 1.2-1.1$ \\
\hline Average pore diameter $(\mu \mathrm{m})$ & $3.0-3.3$ & $3.1-3.2$ & $3.0-3.3$ & $3.0-3.3$ \\
\hline Total porosity (\%) & $30-38$ & $31-32$ & $30-38$ & $30-38$ \\
\hline Thickness of dense PDMS surface layer $(\mu \mathrm{m})$ & - & - & $5-6$ & $10-12$ \\
\hline
\end{tabular}

\footnotetext{
${ }^{\text {a }}$ Pervaporation module was shorter than SGMD module.
} 
A

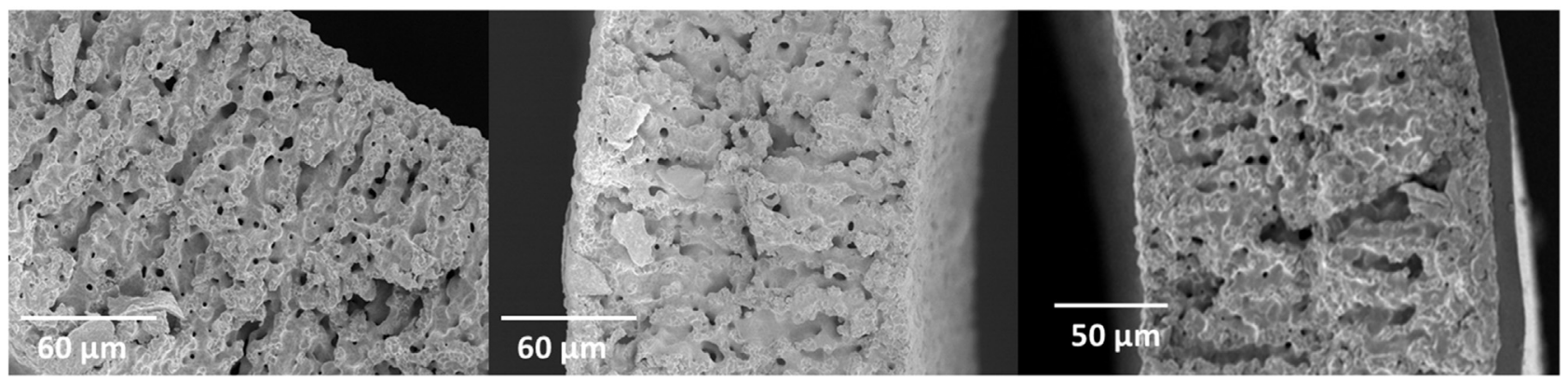

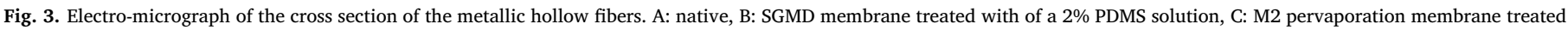
with a $20 \%$ PDMS solution, presenting a thin layer of dense PDMS on its external surface.

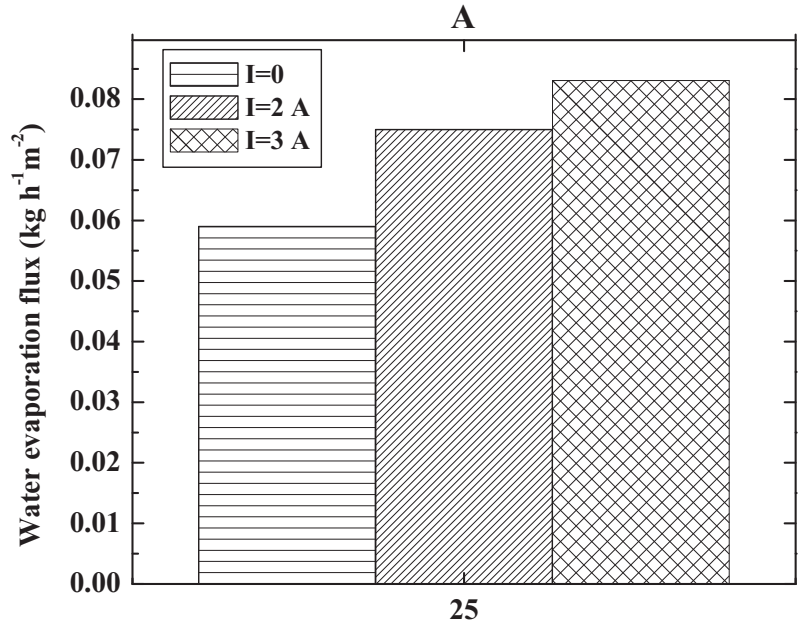

Feed temperature $\left({ }^{\circ} \mathrm{C}\right)$

B

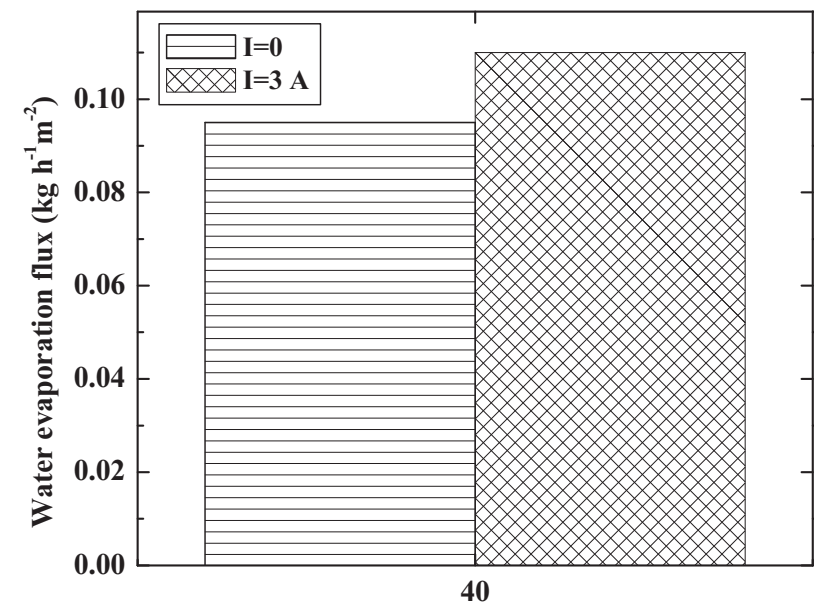

Feed temperature $\left({ }^{\circ} \mathrm{C}\right)$

Fig. 4. Effect of membrane heating on water evaporation fluxes at feed temperatures of $25{ }^{\circ} \mathrm{C}(\mathrm{A})$ and $40{ }^{\circ} \mathrm{C}(\mathrm{B}) .\left(q_{\text {air }}: 60 \mathrm{~L} \mathrm{~h}^{-1}\left(R e_{\text {air }}=1092\right) ; q_{\mathrm{w}}: 34 \mathrm{~L} \mathrm{~h}^{-1}\left(R e_{\text {water }}=145\right) ; \mathrm{T}_{\text {air }}\right.$ : $25 \pm 2{ }^{\circ} \mathrm{C}$; $\mathrm{P}_{\text {air }}$ : atmospheric; standard deviation of evaporation flux $= \pm 0.01 \mathrm{~kg} \mathrm{~h}^{-1} \mathrm{~m}^{-2}$ ).

water evaporation is much lower which could explains the better performances of the directly heated membrane.

Similar effects were observed with experiments using a feed at $40{ }^{\circ} \mathrm{C}$ with flux increasing from 0.095 to $0.110 \mathrm{~kg} \mathrm{~h}^{-1} \mathrm{~m}^{-2}$ at an electrical current of $3 \mathrm{~A}$, corresponding to a flux enhancement of 1.1. It is clear from the above results that the effect of evaporation by membrane heating is more pronounced at low feed temperatures than at high feed temperatures. Furthermore, it is important to state that when the electrical power was shut off, a decline in flux was observed. The decrease was more rapid when the applied power was lower $(I=2 \mathrm{~A})$, whereas the flux remained stable for $I=3 \mathrm{~A}$ at the highest achieved value for a duration of about $30 \mathrm{~min}$ even after shutting off the power, then declined quickly. This testifies the fact that the heating of the membranes could allowed supplying the necessary energy to compensate the loss of flux caused by temperature polarization.

Another important factor which needs to be studied during these experiments is the effect of membrane heating on the feed temperature. To verify whether the energy supplied by the Joule effect does not result in heating of the feed solution, the average water temperature at the outlet of the membrane module was measured in experiments with $(11.7 \mathrm{~W})$ and without $(0 \mathrm{~W})$ electrical input. The obtained values are reported in Table 2.

These data show that the heating system caused an increase in temperature at the outlet of the membrane module of 0.6 and $0.4{ }^{\circ} \mathrm{C}$ for each value of temperature input tested. There is thus an important warming of the entire feed solution.

As far as an important increase of the feed temperature is observed when the electric energy is applied, we analyzed the theoretical increase of water vapor flux reported in Fig. 4A, when we supply $12 \mathrm{~W}$ of electrical energy (from $0 \mathrm{~A}$ to $3 \mathrm{~A}$ ). Indeed the experimental water vapor flux enhancement observed is of $2.410^{-2} \mathrm{~kg} / \mathrm{h}^{-1} \mathrm{~m}^{2}$.

In one hand, for the theoretical analysis we have to consider the enthalpy used for the increase in water vapor flux, which is given by:

$\Delta J$. A. $\Delta H_{v}$

where $\Delta J=$ is the flux increase, $\mathrm{kg} / \mathrm{m}^{2} \mathrm{~s}, A$ is the total membrane area $\left(\mathrm{m}^{2}\right)$ and $\Delta H_{v}$ is the heat of evaporation of water $(\mathrm{J} / \mathrm{kg})$.

Then, we have $\Delta J=\left(2.4 \times 10^{-2} / 3600\right)\left(1.4 \times 10^{-2}\right)\left(2257 \times 10^{3}\right)$ $=0.211 \mathrm{~W}$

In other words only $1.8 \%$ of the electric energy is used for the vaporization of water.

On the other hand, the electric energy necessary for the feed water heating is:

$C_{p} . F . \Delta T$

Table 2

Steady state water temperature at the outlet of membrane module when tested with and without Joule heating (Feed: pure water; $R \mathrm{e}_{\text {water }}=145 ; R e_{\text {air }}=1092 ; \mathrm{P}_{\text {air }}=$ atmospheric; $\mathrm{T}_{\text {air }}=25 \pm 2{ }^{\circ} \mathrm{C}$ ).

\begin{tabular}{|c|c|c|c|c|}
\hline $\begin{array}{l}\text { Temperature of the feed solution at the module } \\
\text { entrance }\left({ }^{\circ} \mathrm{C}\right)\end{array}$ & 25.6 & & 40.0 & \\
\hline Heating system & off & on & Off & on \\
\hline $\begin{array}{l}\text { Temperature of the feed solution at the module exit } \\
\left({ }^{\circ} \mathrm{C}\right)\end{array}$ & 25.4 & 26.0 & 40.5 & 40.9 \\
\hline
\end{tabular}

Accuracy of sensors: $\pm 0.3{ }^{\circ} \mathrm{C}$. 
Table 3

Experimental conditions for pervaporation.

\begin{tabular}{lll}
\hline Membrane & M1 & M2 \\
\hline \% PDMS coating & 10 & 20 \\
wt\% Ethanol concentration in feed & $2,5,10,20$ & $5,10,20$ \\
Applied current I (A) (4 V voltage) & 0,4 & 0,4 \\
\hline
\end{tabular}

where $C_{p}$ is heat capacity of water $(\mathrm{J} / \mathrm{kg} \mathrm{K}), F$ is feed flow rate $\left(\mathrm{m}^{3} / \mathrm{s}\right)$ and $\Delta T$ is the increase in the feed temperature (K).

Using $C_{p}=4.18 \times 10^{3} \mathrm{~J} / \mathrm{kg} \mathrm{K}$ (at $25^{\circ} \mathrm{C}$ ) and $12 \mathrm{~W}$ of electrical energy input, then

$$
\text { F. } \begin{aligned}
\Delta T & =(12) /\left(4.18 \times 10^{3}\right)=2.87 \times 10^{-3}(\mathrm{~kg} \mathrm{~K} / \mathrm{s})=2.87 \times 10^{-3} \mathrm{~L} \mathrm{~K} / \mathrm{s} \\
& =0.172 \mathrm{~L} \mathrm{~K} \mathrm{~min}^{-1}
\end{aligned}
$$

This means that for the flow rate of $0.56 \mathrm{~L} \mathrm{~min}^{-1}\left(34 \mathrm{~L} \mathrm{~h}^{-1}\right.$ from Ref. [12]) the increase in feed temperature is $0.31{ }^{\circ} \mathrm{C}$.

Since the reported values of increase in the feed temperature are $0.4-0.6{ }^{\circ} \mathrm{C}$, we can conclude that most of electrical energy was used for heating the feed. Indeed, the water vapor flux enhancement observed is certainly caused by the decrease of the temperature polarization phenomenon at the vicinity of the membrane level but also by the enhancement of the feed temperature.

\subsection{Pervaporation}

As explained above, pervaporation experiments were carried out with membranes presenting a dense layer of PDMS on the surface of the porous metallic hollow fibers: Table 3 gives a brief overview of membranes, feed compositions and process variables employed in these experiments.

Two sets of experiments, with and without applying electricity, using both membranes (M1 and M2) at the respective feed compositions mentioned in Table 3 were conducted. The maximum value of current used to heat the membrane was fixed to $4 \mathrm{~A}$.

The results were reproducible with a deviation of \pm 0.001 $\mathrm{kg} \mathrm{h}^{-1} \mathrm{~m}^{-2}$ for flux. Fig. 5 presents results of the pervaporation with a $10 \%$ PDMS coated membrane (M1). The membrane performance in terms of pervaporation flux and separation factor in absence and presence of electricity is presented in Fig. 5A and B respectively.

As expected, the general trend is that with increased ethanol concentration in the feed solution, the flux increases and the separation factor decreases. A pronounced increase in the flux is observed when the membrane is electrically heated. Similar behavior was seen when experiments were performed with $20 \%$ PDMS coated membrane (M2), represented in Fig. 6A and B.

Basic understanding of the mass transfer across the membrane during pervaporation by the solution-diffusion mechanism offers an explanation for these trends observed with both membranes. During pervaporation, selective sorption of ethanol into the PDMS layer happens because of its higher affinity towards PDMS. In the next step, ethanol molecules diffuse towards the permeate side where they are desorbed as a vapor. Affinity between water and the selective layer is lower owing to the hydrophobicity of PDMS. When the ethanol concentration in the feed increases, the overall sorption of ethanol into PDMS also increases, resulting in swelling of the PDMS network. The reduced transport resistance because of the swollen selective layer causes an increased flux. The decline in selectivity can be explained by thermodynamic interactions between the components in a liquid mixture [16]. According to this principle, the membrane becomes accessible for another component (in this case water) which otherwise has a lower permeability in PDMS, because of increased interaction of ethanol with PDMS and its resulting swelling. This explains the decreased separation factor with increasing ethanol concentration. It can be noticed that in experiments with membrane M1 (Fig. 5), the
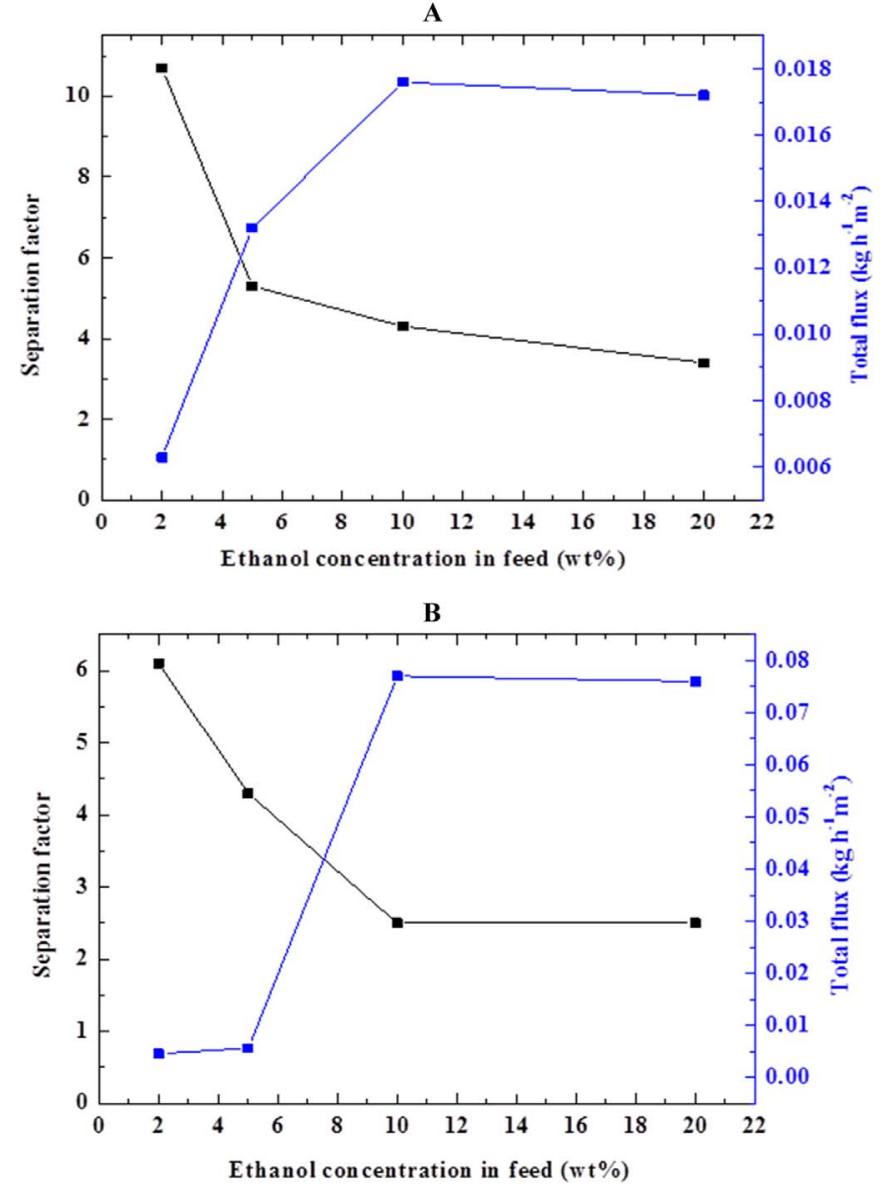

Fig. 5. Comparison of pervaporation performance of 10\% PDMS hollow fiber coated membrane (M1) without (A) and with (B) electrical heating: (Feed: Ethanol (2-20 wt $\%$ in

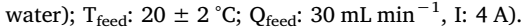

separation factor and flux are almost stabilized after $10 \%$ wt ethanol in the feed. This result could be explained by the fact that the thin PDMS layer membrane $(5-6 \mu \mathrm{m})$ is already saturated with ethanol. This is not the case for the thicker membrane M2 (10-12 $\mu \mathrm{m})$ which is still able to sorb the organic compound.

M2 membranes yielded lower fluxes than the M1 membranes for experiments with and without electricity. This is due to the thicker PDMS layer in the case of M2 which was about 10-12 $\mu \mathrm{m}$; while 5-6 $\mu \mathrm{m}$ for M1. By electrical heating of the membrane, a very clear rise in flux was observed for both membranes. The highest achieved flux at maximum ethanol concentration (20 wt\%) was 0.08 and $0.012 \mathrm{~kg} \mathrm{~h}^{-1} \mathrm{~m}^{-2}$ for M1 and M2 respectively, which is almost four times the fluxes obtained without electricity.

In terms of separation, M1 exhibited better separation factor values than M2. The highest separation factors obtained in this study were obtained using M1, i.e. $6(I=0 \mathrm{~A})$ and $10(I=4 \mathrm{~A})$ for $2 \mathrm{wt} \%$ feed ethanol concentrations. The separation abilities of both membranes can be compared at the same feed ethanol concentration. For example, at an ethanol feed concentration of $20 \mathrm{wt} \%$, the separation factors improved in the case of M2 from 1.4 to 3 upon applying electricity to the membrane. Similar for M1, the increase was from 2.5 to 4 upon membrane heating. In both cases, the factors are thus almost doubled. If we compare both membranes M1 and M2, It is difficult to give clear explanations about the increase of the selectivity with the decrease of the membrane thickness without electric heating and at same ethanol content. Normally for "defaults free membranes" prepared with the same PDMS we have to observe the same selectivity but decreasing fluxes with the thickness enhancement. This behavior normalizes when electric heating is applied because both types of membranes present the 


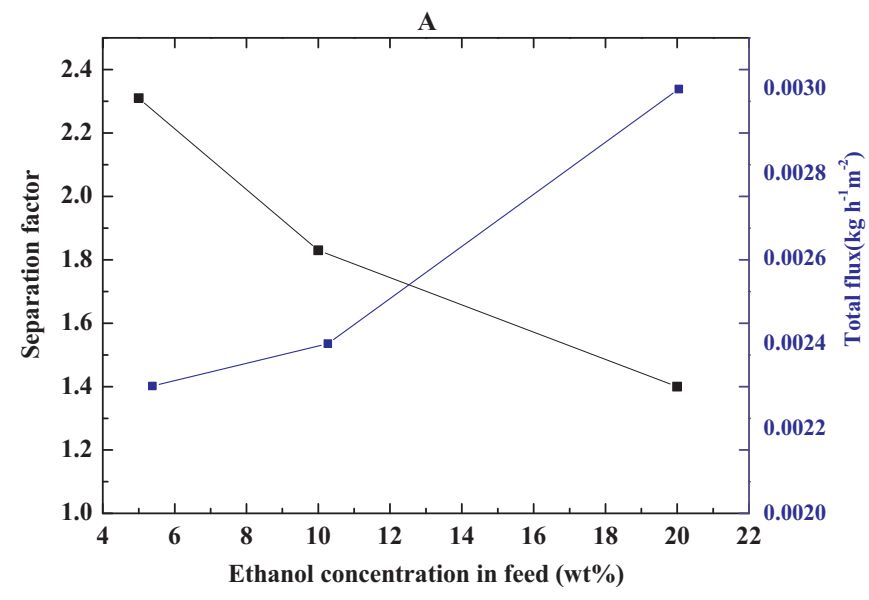

B

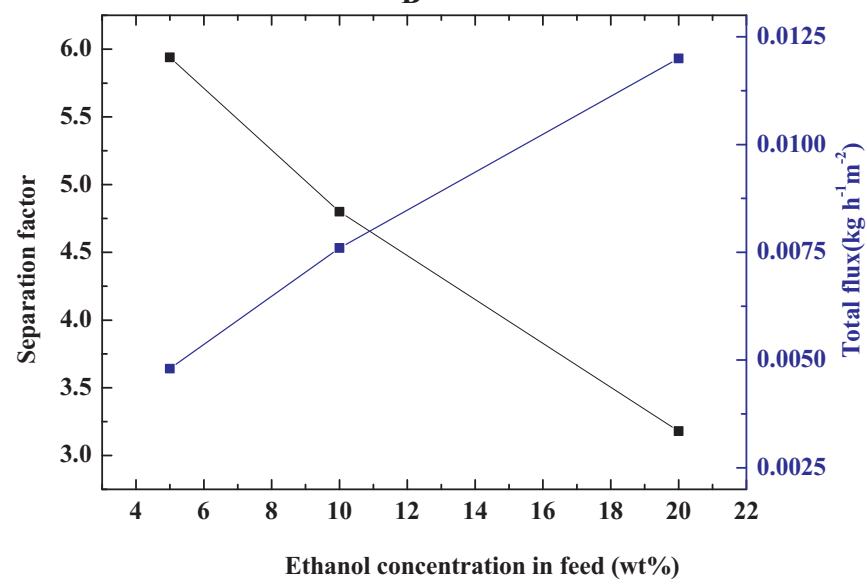

Fig. 6. Comparison of pervaporation performance of 20\% PDMS hollow fiber coated membrane (M2) without (A) and with (B) and electrical heating (Feed: Ethanol (2-20 wt $\%$ in water); $\mathrm{T}_{\text {feed }}: 20 \pm 2{ }^{\circ} \mathrm{C}$; $\mathrm{q}_{\text {feed }}: 30 \mathrm{~mL} \mathrm{~min}^{-1}$, I: $4 \mathrm{~A}$ ).

same order of selectivity at the same ethanol content when heating is applied.

The improvement of the membrane flux can be explained by the fact that, when electricity is applied, the metallic support heats up. In conventional pervaporation processes, heating the feed is commonly practiced to supply the enthalpy of vaporization. However, with the evaporation of the permeating compounds, some part of the heat present at the feed side is lost for vaporization during transport through the membrane, hence generating a temperature profile. The temperature is thus maximal in the bulk feed and lower in the selective layer and lowest in the membrane support. Under such circumstances, vapor recondensation can occur in the support or at the bottom of the selective layer which would reduce the membrane flux. It is this loss of energy that can be made up with membrane heating. Higher temperatures can be maintained in the membrane and in the support, thus avoiding vapor re-condensation problems while increasing the diffusion of ethanol. During the experiment, the temperature increase on the membrane surface was monitored for experiments with and without electricity. It was observed that the temperature on the membrane surface was always around $27 \pm 2{ }^{\circ} \mathrm{C}$ with electricity, with the feed maintained initially at temperatures of $21-22^{\circ} \mathrm{C}$.

The increase in separation factor upon membrane heating can be explained in terms of enthalpy of vaporization required to evaporate the feed components. The enthalpy of vaporization for ethanol is lower than that of water, respectively 38.6 and $40.68 \mathrm{~kJ} \mathrm{~mol}^{-1}$ at standard conditions. The PDMS layer is almost saturated with ethanol due to its preferential affinity for ethanol than water. Thus with membrane heating, ethanol molecules are preferably evaporated more than water, enhancing the separation factor.

As mentioned before, there are only few studies which adapted the approach of heating the membrane instead of the feed in pervaporation. Komorowska-Durka et al. [13] employed microwave heating of membranes in the dehydration of water from water-ethanol mixtures across a PVA-cross-linked PAN membrane. The authors reported that in the feed at $5.5 \mathrm{wt} \%$ water, the total flux was higher under microwave heating than conventional heating. At a feed temperature of $33.5^{\circ} \mathrm{C}$, which is slightly higher than the feed temperatures in our study, the authors were successful in increasing the water flux from $2.010^{-2}$ to $4.010^{-2} \mathrm{~kg} \mathrm{~h}^{-1} \mathrm{~m}^{-2}$ on replacing feed heating by membrane heating with microwave. Though this study concerns hydrophilic pervaporation, the principle is the same and so are the observations. In our study, permeate fluxes are slightly lower $\left(0.012 \mathrm{~kg} \mathrm{~h}^{-1} \mathrm{~m}^{-2}\right.$ at $5 \mathrm{wt} \%$ ethanol concentration) but the temperature required in the feed is also lower which is better for thermally sensitive component and seems a promising improvement.

Li et al. [7] made membranes with silver nanoparticles embedded in 20\% PDMS membranes supported over PVDF. Analogous to our observation, they also observed an increase in flux and selectivity on heating the membrane with a LED light source. The highest flux and selectivity under optimized feed flow rates were $0.160 \mathrm{~kg} \mathrm{~h}^{-1} \mathrm{~m}^{-2}$ and 3 respectively. In our case, a separation factor of 6 was obtained under the influence of electricity at the same $5 \mathrm{wt} \%$ feed ethanol concentration. However direct comparison of flux is difficult with insufficient information of selective layer thickness provided in the reference study.

\section{Conclusions}

The proof of concept was given of membrane process intensification by coupling direct membrane heating to SGMD and pervaporation. Electrical heating of hydrophobic metallic hollow fibers could improve water vapor fluxes in SGMD, as the result of two phenomena: the decrease of the heat lost by evaporation by a direct supply of energy by the Joule effect right at the membrane pores and the improvement of the feed temperature. Water vapor fluxes are enhanced under an electrical potential up to $40 \%$.

This simple way to supply energy could be easily adapted to a hollow fiber configuration. The obtained increase in flux is promising because a fraction of the energy supplied is affectively used for compensating the heat lost by evaporation, even if the majority of the energy is consumed to heat the solution to be concentrated. Nevertheless, the heating of the feed solution is relatively low (only $0.4-0.6{ }^{\circ} \mathrm{C}$ ) and the process seems to be potentially interesting for the concentration of thermally sensitive solutions.

The use of metallic hollow fibers for SGMD application needs further improvements because the water vapor fluxes reached are still modest when compared with polymeric hollow fibers. Improving the porosity of these membranes alongside with keeping their good mechanical resistance will be fundamental for further developments.

Improvement in hydrophobic pervaporation performance in terms of both flux and separation factor has been established. Although these flux and selectivity improvements are lower compared to feed heating (as per literature), they can be further improved by working on increasing the support porosity and decreasing the thickness and they also require much less energy input. More investigations are underway to study the effect of varying strengths of the electric current on pervaporation performance and fine-tuning the PDMS selective layer to achieve the best possible results. In addition, the study will be completed by the development of 2D and 3D models including CFD, in order to simulate the SGMD process and optimize the geometry of the module as well as the structural characteristics of the membranes.

\section{Acknowledgments}

S. Shukla thanks the European Commission - Education, Audiovisual 
and Culture Executive Agency (EACEA), under the program: Erasmus Mundus Doctorate in Membrane Engineering - EUDIME (FPA N ${ }^{\circ} 2011$ 0014, Edition I, http:/eudime.unical.it). We are also grateful to KU Leuven for support in the frame of IOF-funding (IKP/10/002) and the Federal Government for an IAP grant (FS2).

\section{References}

[1] M. Khayet, Membranes and theoretical modeling of membrane distillation: a review, Adv. Colloid Interface Sci. 164 (2011) 56-88.

[2] P. Wang, T.S. Chung, Recent advances in membrane distillation processes: membrane development, configuration design and application exploring, J. Membr. Sci. 474 (2015) 39-56.

[3] (a) L.M. Camacho, L. Dumée, J. Zhang, J. Li, M. Duke, J. Gomez, S. Gray, Advances in membrane distillation for water desalination and purification applications, Water 5 (2013) 94-196A;

(b) Gabelman, S. Hwang, Hollow fiber membrane contactors, J. Membr. Sci. 159 (1999) 61-106.

[4] (a) R.W. Baker, Membrane Technology and Applications, Second ed., John Wiley \& Sons Ltd, England, 2004;

(b) E. Curcio, E. Drioli, A. Criscuoli, Membrane Contactors: Fundamentals, Applications and Potentials, 11th ed., Elsevier, 2005.

[5] B. Smitha, D. Suhanya, S. Sridhar, M. Ramakrishna, Separation of organic-organic mixtures by pervaporation - a review, J. Membr. Sci. 241 (2004) 1-21.

[6] Y. Li, T. Verbiest, R. Strobbe, I.F.J. Vankelecom, Silver nanoparticles as localized "nano-heaters" under LED light irradiation to improve membrane performance, J. Mater. Chem. A 2 (2014) 3182.

[7] Y. Li, T. Verbiest, R. Strobbe, I.F.J. Vankelecom, Improving the performance of pervaporation membranes via localized heating through incorporation of silver nanoparticles, J. Mater. Chem. A 1 (2013) 15031-15038.

[8] Y. Li, T. Verbiest, I. Vankelecom, Improving the flux of PDMS membranes via localized heating through incorporation of gold nanoparticles, J. Membr. Sci. 428 (2013) 63-69.

[9] N. Hengl, A. Mourgues, E. Pomier, M.P. Belleville, D. Paolucci-Jeanjean, J. Sanchez, G. Rios, Study of a new membrane evaporator with a hydrophobic metallic membrane, J. Membr. Sci. 289 (2007) 169-177.

[10] N. Hengl, A. Mourgues, M.-P. Belleville, D. Paolucci-Jeanjean, J. Sanchez, Membrane contactor with hydrophobic metallic membranes: 2. Study of operating parameters in membrane evaporation, J. Memb. Sci. 355 (2010) 126-132.

[11] (a) A. Mourgues, N. Hengl, M.P. Belleville, D. Paolucci-Jeanjean, J. Sanchez, Membrane contactor with hydrophobic metallic membranes: 1 . Modeling of coupled mass and heat transfers in membrane evaporation, J. Membr. Sci. 355 (2010) 112-125;

(b) M. Khayet, P. Godino, J.I. Mengual, Theory and experiments on SGMD, J. Memb. Sci. 165 (2000) 261-272.

[12] Shukla, N.E. Benes, I. Vankelecom, J.P. Méricq, M.P. Belleville, N. Hengl, J. Sanchez Marcano, Sweep gas membrane distillation in a membrane contactor with metallic hollow-fibers, S. J. Membr. Sci. 493 (2015) 167-178.

[13] M. Komorowska-Durka, R. van Houten, G.D. Stefanidis, Application of microwave heating to pervaporation: a case study for separation of ethanol-water mixtures, Chem. Eng. Process.: Process Intensif. 81 (2014) 35-40.

[14] N. Kikuo, O. Shinichi, Hollow-fiber Membranes for Pervaporation Separation Applied in Electromagnetic Microwave Field, Agency of Industrial Sciences and Technology, 1985.

[15] S.M. Dutczak, M.W.J. Luiten-Olieman, H.J. Zwijnenberg, L.A.M. Bolhuis-Versteeg, L. Winnubst, M.A. Hempenius, N.E. Benes, M. Wessling, D. Stamatialis, Composite capillary membrane for solvent resistant nanofiltration, J. Membr. Sci. 372 (2011) 182-190.

[16] M. Mulder, Basic Principles of Membrane Technology, 2nd ed., Kluwer Academic publishers, Dordrecht, 1996. 